\title{
Patterning of light-emitting conjugated polymer nanofibres
}

\author{
FRANCESCA DI BENEDETTO ${ }^{1}$, ANDREA CAMPOSE0 ${ }^{1}$, STEFANO PAGLIARA', ${ }^{1,2}$ ELISA MELE¹, \\ LUANA PERSAN0 ${ }^{1}$, RIPALTA STABILE ${ }^{1,2}$, ROBERTO CINGOLANI ${ }^{1}$ AND DARIO PISIGNAN01,2*
}

${ }^{1}$ Italian Institute of Technology, Research Unit at NNL, National Nanotechnology Laboratory of INFM-CNR, via Arnesano, I-73100 Lecce, Italy ${ }^{2}$ Scuola Superiore ISUFI, Università del Salento, via Arnesano, I-73100 Lecce, Italy

*e-mail: dario.pisignano@unile.it

Organic materials have revolutionized optoelectronics by their processability, flexibility and low cost, with application to lightemitting devices for full-colour screens ${ }^{1}$, solar cells ${ }^{2}$ and lasers $^{3,4}$. Some low-dimensional organic semiconductor structures exhibit properties resembling those of inorganics, such as polarized emission ${ }^{5}$ and enhanced electroluminescence ${ }^{6}$. One-dimensional metallic, III-V and II-VI nanostructures have also been the subject of intense investigation ${ }^{7,8}$ as building blocks for nanoelectronics and photonics. Given that onedimensional polymer nanostructures, such as polymer nanofibres, are compatible with sub-micrometre patterning capability $^{9}$ and electromagnetic confinement within subwavelength volumes ${ }^{8}$, they can offer the benefits of organic light sources to nanoscale optics. Here we report on the optical properties of fully conjugated, electrospun polymer nanofibres. We assess their waveguiding performance and emission tuneability in the whole visible range. We demonstrate the enhancement of the fibre forward emission through imprinting periodic nanostructures using room-temperature nanoimprint lithography, and investigate the angular dispersion of differently polarized emitted light.

Nanopatterning and internal waveguiding of nanophotonic sources remain challenging. For instance, light confinement in polymers may suffer due to their low refractive index, $n$. For this reason, organic miniaturized optical fibres are generally based on passive high- $n$ resists, possibly incorporating inorganic quantum dots $^{10}$. In addition, light-emitting organics require specific, often technologically demanding and low-throughput nanolithography tools to be patterned at sub-micrometre resolution without oxygen-induced alteration of their emissive properties.

In contrast to other methods ${ }^{11-13}$, electrostatic spinning permits processing of large volumes of polymers and the formation of fibres with sub-micrometre diameter by means of plastic stretching ${ }^{14-17}$, and importantly promotes internal molecular orientation of the organic molecules by means of the applied electric field ${ }^{18}$. Because of the limited solubility and relatively poor viscoelastic behaviour of conjugated polymers, proposed approaches for performing electrostatic spinning of such materials ${ }^{15,19-24}$ rely on blends or core-shell heterostructures with more solutionprocessable but optically inert compounds. However, although conjugated polymers tend to gel at high solution concentrations, we saw that using non-solvents with a high boiling point and dielectric constant ${ }^{25}$ improves their processability, facilitating the realization of single-component conjugated polymer fibres using electrostatic spinning. Also, the polymer side-groups can increase the steric hindrance and the conformational plasticity of viscous solutions. Here, we make several different conjugated polymer fibres using electrostatic spinning (the molecular structures of the polymers used are shown in Fig. 1a) and use room-temperature nanoimprint lithography (RT-NIL) to pattern them. We find that the polymer fibre section varies from the micrometre scale down to tens of nanometres and is controllable by adjusting the electrostatic spinning bias (Fig. 1b,c). Figure 1d shows the photoluminescence spectra of as-produced fibres, which cover the whole visible range and are stable for several months under ambient storage conditions.

The fluorescence micrographs of the fibres (Fig. 1e-h) also show evidence of waveguiding capability. To have a better insight into this issue, propagation loss measurements are performed by collecting the PL signal from the tip of single nanofibres, excited by a tightly focused laser beam (Fig. 2a). For poly[(9,9-dioctylfluorenyl2,7-diyl)-co-(1,4-benzo- $\left\{2,1^{\prime}, 3\right\}$-thiadiazole)] (YE) fibres spun from dimethyl sulphoxide and tetrahydrofuran with 1:15 volume:volume relative concentration, the photoluminescence decreases as a function of the distance from the exciting laser spot, providing a loss coefficient $\gamma$ of $3 \times 10^{2} \mathrm{~cm}^{-1}$ (Fig. 2b), which is lower than the values reported for semicrystalline polyfluorene nanowires ${ }^{26}$. The optical losses measured here can be linked to polymer self-absorption, and to scattering from the fibre bulk inhomogeneities or surface defects. The losses are significantly lower than those evaluated from the spectral overlap between the absorption and emission of the conjugated molecule in thin-film form $\left(>2 \times 10^{3} \mathrm{~cm}^{-1}\right.$; see inset, Fig. $\left.2 \mathrm{~b}\right)$. Such a reduced nanofibre self-absorption is likely related to the preferential supramolecular organization and orientation of the polymer backbones induced by the electrostatic spinning procedure ${ }^{18}$. In addition, possible local regions with non-uniform refractive index, density or crystallinity produce bulk scattering in the lightemitting fibres. For instance, electrostatic spinning of YE by tetrahydrofuran only provides fibres with inhomogeneities with linear density of $\sim 500-800$ scattering centres per centimetre and typical size of between 10 and $100 \mathrm{~nm}$ (see inset, Fig. 2c). These odd optical domains affect the resulting emission, whose intensity exhibits abrupt discontinuities along the fibre axis and higher local optical losses (Fig. 2c), whereas simultaneously using dimethyl sulphoxide and tetrahydrofuran as electrostatic spinning solvents 
a

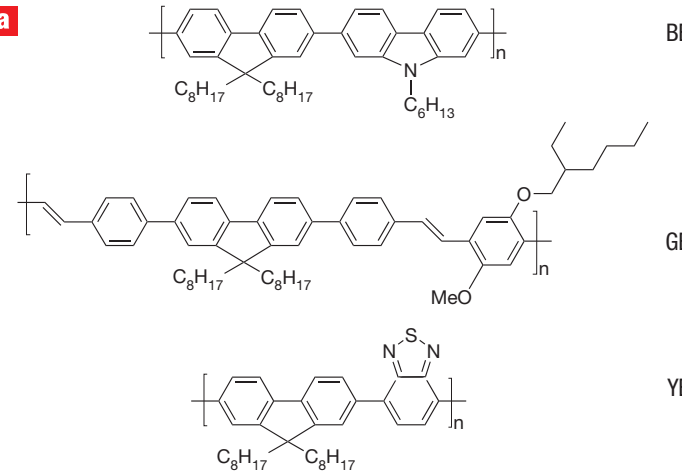<smiles>CCCCC(CC)COc1cc(C=Cc2cc(C)cc(C)c2)c(O)cc1C=Nc1ccc(C)cc1</smiles>

MEH-PPV

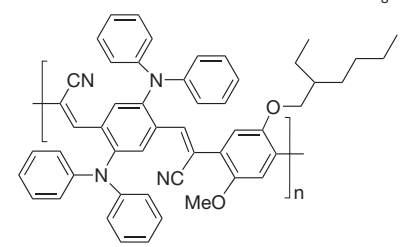

d

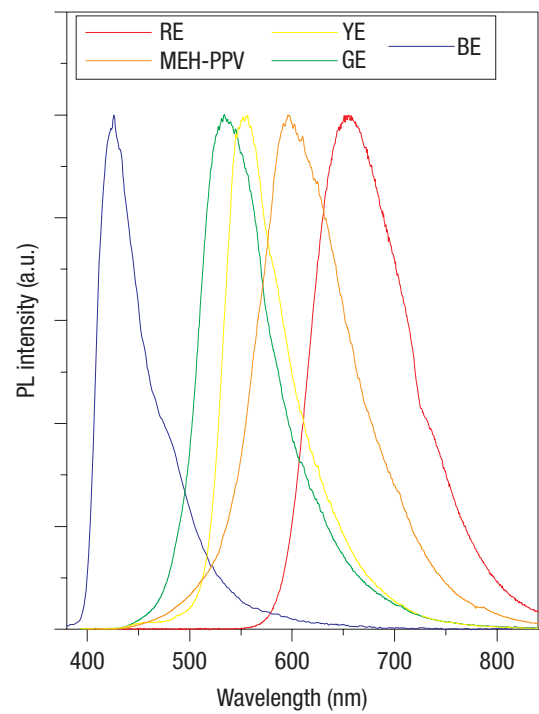

$\mathrm{BE}$

GE

YE

RE

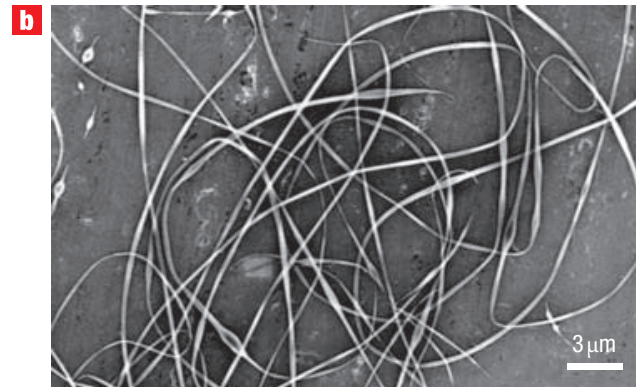

G

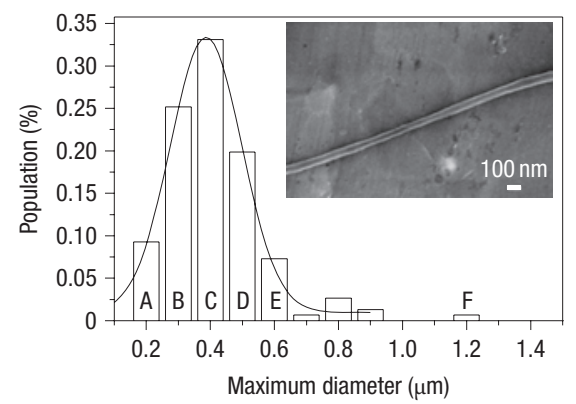

e

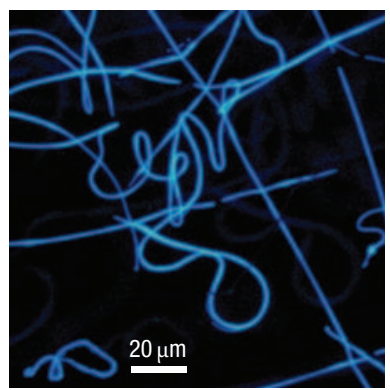

g

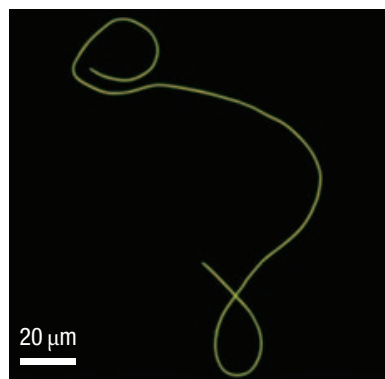

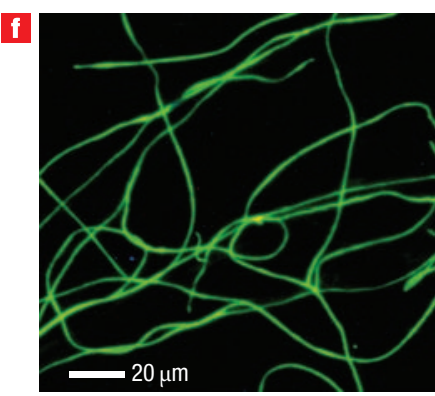

h

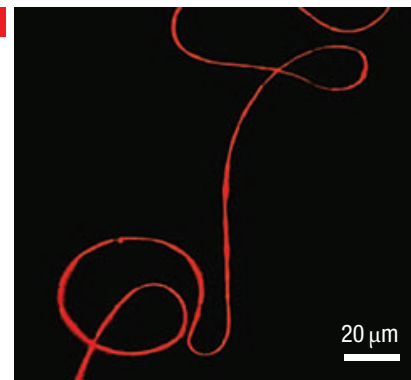

Figure 1 Characterization and optical properties of as-produced conjugated polymer nanofibres. a, Molecular structures of the polymers.

Poly[(9,9-dioctylfluorenyl-2,7-diyl)-alt-co-(9-hexyl-3,6-carbazole)] (BE), poly[(9,9-dioctylfluorenylene-2,7-diyl)-co-(1,4-diphenylenevinylene-2-methoxy5-\{2-ethylhexyloxy\}-benzene)] (GE), poly[(9,9-dioctylfluorenyl-2,7-diyl)-co-(1,4-benzo- $\left\{2,1^{\prime}, 3\right\}$-thiadiazole)] (YE), poly[2-methoxy-5-(2-ethylhexyloxy)1,4-phenylenevinylene] (MEH-PPV) and poly[\{2-methoxy-5-(2-ethylhexyloxy\}-1,4-(1-cyanovinylenephenylene))-co- $\{2,5$ - bis(N,N'-diphenylamino)-1,4-phenylene $\}]$ (RE). b. SEM image of conjugated polymer nanofibres (RE from 1:5 dimethyl formamide:chloroform solutions). c, Typical diameter (S) distribution and fit by a Gaussian curve (solid line). The letters indicate groups of fibres with similar diameters (see Methods). Inset: RE fibre with a diameter of $75 \mathrm{~nm}$. d, Normalized photoluminescence spectra from nanofibre mats. e-h, Fluorescence micrographs of emitting fibres for polymers BE (e), GE (f), YE (g) and RE (h), respectively.

allows homogeneous YE fibres without microscopically appreciable scattering domains to be obtained. These results highlight the importance of the solvents in affecting the internal waveguiding performance in conjugated polymer fibres.

We also investigated the influence of the substrate material on waveguiding by measuring the optical losses on fibres with the same diameter and composition (YE from dimethyl sulphoxide:tetrahydrofuran), deposited on glass $(n=1.51)$ and silicon $(n=3.87)$. We can detect slight differences in the waveguide performance (Fig. 2d), measuring losses of $5 \times 10^{2} \mathrm{~cm}^{-1}$ and $7 \times 10^{2} \mathrm{~cm}^{-1}$ for the low- $n$ and high- $n$ substrates, respectively, which is evidence of a coupling of the guided radiation to the substrate due to the rounded shape of the fibre and the limited contact with the underlying material. This can 

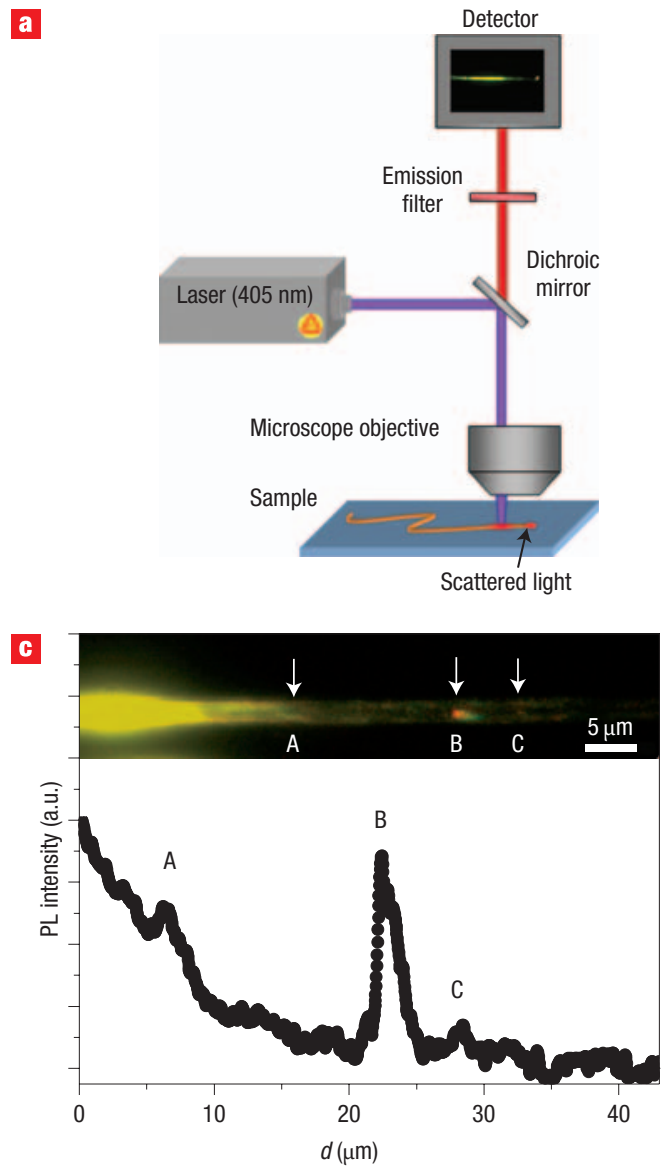

b
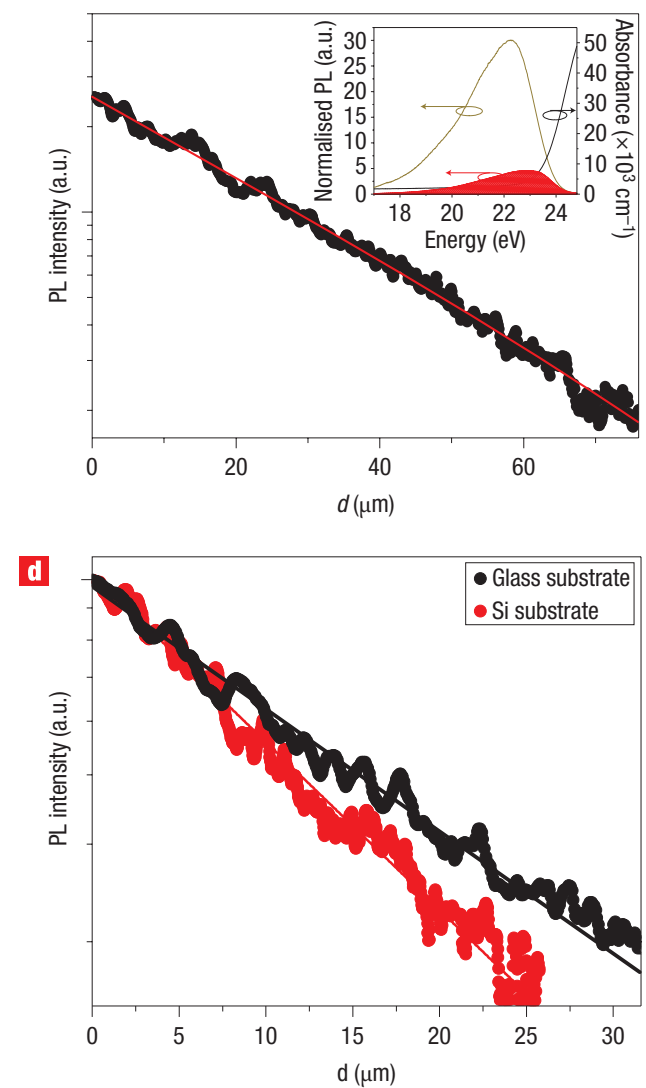

Figure 2 Nanofibre waveguiding properties. a, Scheme of the experimental setup used for characterizing single-fibre waveguiding. $\mathbf{b}$, Dependence of the intensity of the guided light inside a single YE fibre, electrospun from 1:15 dimethyl sulphoxide:tetrahydrofuran solution, on distance $d$, measured as the distance from a photo-excited region to the collection point. The superimposed continuous line is the best fit of the data to the equation $I=I_{0} \exp (-\gamma d)$. Inset: YE thin-film photoluminescence intensity (yellow spectrum) and absorbance (black spectrum), together with the overlap spectrum (=photoluminescence $\times$ absorbance, red line), whose integral determines the polymer self-absorption. The YE thin-film was spin-cast from the same solution as used for electrostatic spinning. c, Dependence of the intensity of the guided light inside a single YE fibre, electrospun from tetrahydrofuran solution, on distance $d$. Inset: corresponding fluorescence micrographs. The three inhomogeneous optical domains are labelled A, B and C, and are indicated by arrows in the inset. d, Dependence of the intensity of the guided light inside a single YE fibre at distance $d$, when deposited on glass (black dots) or silicon (red dots). The superimposed lines represent best fits to the equation $I=I_{0} \exp (-\gamma d$ ).

be advantageous, as it allows waveguided light to be coupled to on-chip detectors for sensing and nanophotonic applications.

For realizing photonic nanostructures based on conjugated polymer nanofibres, we made use of their processability by patterning using RT-NIL ${ }^{27}$. RT-NIL is a high-throughput technique based on the deformation of an organic layer under the application of pressure by a rigid nanostructured template, below the glass transition temperature, and without exposure to beams or solvents, surface treatments or a controlled atmosphere. In this way, RT-NIL overcomes the limitations of hot embossing for directly structuring conjugated molecules in air, as the optoelectronic properties of the active material do not undergo irreversible degradation due to oxygen incorporation into the molecular backbones. Such an approach would enable the realization of imprinted distributed feedback devices, which rely on periodic, wavelength-scale variation of optical gain or refractive index along the fibres.

We patterned single fibres with a resolution up to the $100 \mathrm{~nm}$ scale (Fig. 3). Because of the high applied pressure (240-290 $\mathrm{MPa}$ ) fibres can undergo partial flattening upon RT-NIL. Once the sample convolution effect resulting from the physical dimension of the atomic force microscope (AFM) tip is taken into account, the fibre height/width reduction after patterning is $<35 \%$ (Fig. 3a,b). As this effect is strictly related to the amount of polymer filling the recessed features of the master during pressure-induced flow, it can be greatly reduced by using masters with features having higher aspect ratios. The plan view and cross-section of on-fibre printed gratings with period in the range $400-640 \mathrm{~nm}$ show very well-defined patterns, the achieved resolution being dependent on the starting masters. The protruding master features determine neat indentations, with angle relative to the fibre axis depending on the mutual master/sample orientation during imprinting. The resulting patterns present the same period of the pressing mould and a feature height up to $150-200 \mathrm{~nm}$ (Fig. 3c-e), without significant reduction in the aspect ratio with respect to the master. By using AFM we established the absence of a bottom layer between indentations along the imprinted fibres. This effect is relevant, because a bottom layer would cause interference or crosstalk between adjacent light-emitting 

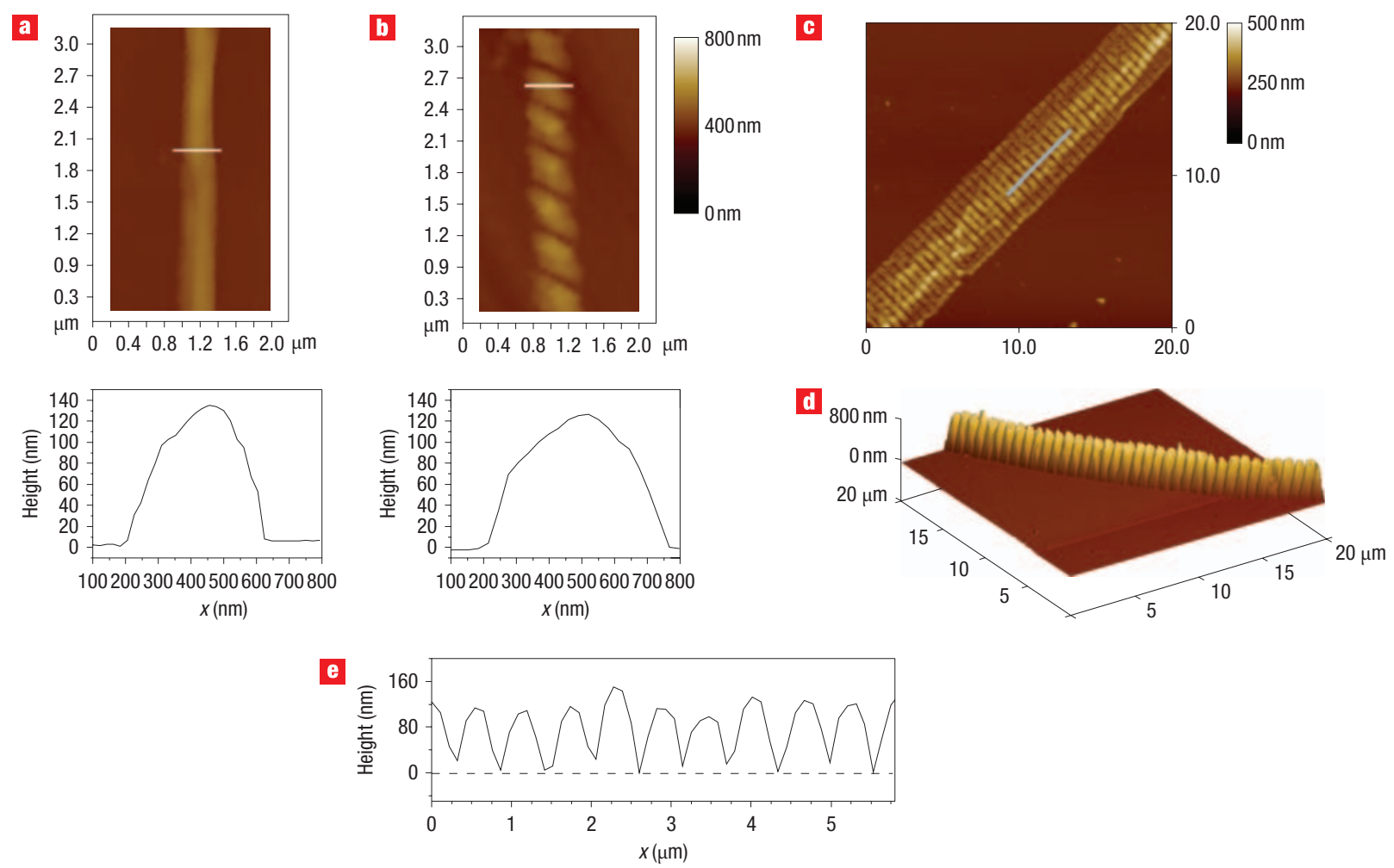

Figure 3 Nanopatterning of single light-emitting polymer nanofibres. a,b, AFM planar views and corresponding cross-sections of an MEH-PPV fibre electrospun from 1:5 dimethyl sulphoxide:tetrahydrofuran, before (a) and after (b) RT-NIL of a 400-nm-period grating (grating/fibre axis angle $\approx 60^{\circ}$ ). The height profiles are taken along the blue lines marking the fibre cross-sections in the planar views. c, AFM planar view $\left(20 \times 20 \mu \mathrm{m}^{2}\right)$ of a 550-nm-period grating (grating/fibre axis angle $\approx 90^{\circ}$ ). $\mathbf{d}$, Three-dimensional imaging of a 640 -nm-period grating on a single fibre. $\mathbf{e}$, The height profile along the blue line marking the fibre axis in $\mathbf{c}$.

features and a decrease in the optical feedback from on-fibre printed microcavities.

In order to assess the influence of patterning on emission, we measured single-fibre, angle-resolved photoluminescence before and after nanolithography. Fibres with diameters intentionally produced to be in the range $0.5-5 \mu \mathrm{m}$ are used to collect reliable fluorescence micrographs and photoluminescence spectra. Confocal microscopy (Fig. 4a) on nanopatterned fibres shows uniformly bright imprinted gratings. The spectra of a typical MEH-PPV fibre are displayed in Fig. 4b. Although MEH-PPV molecules ${ }^{28}$ and films ${ }^{4}$ often present $S_{1}-S_{0}$ transitions with a $0-0$ vibronic replica more intense than the $0-1$ replica, in the emission of the untextured fibre the two replicas exhibit almost the same relative intensity, that is, a relative decrease of the high-energy spectral component $(\lambda<600 \mathrm{~nm})$. This lineshape is affected by chain packaging morphology ${ }^{4}$ and by internal waveguiding in the fibres, which is more effective at low wavelengths, thus decreasing the intensity of the externally coupled light.

Upon patterning, we find clear evidence for an enhancement of the nanofibre forward emission, namely of the light collected at angles $\Theta \leq 70^{\circ}$ as the detection is moved perpendicularly to the direction of the patterned grooves. This increases the overall light output from the fibre source; that is, it enhances the external quantum efficiency of the light-emitting nanostructure, because it reduces the effective length covered by the emitted photons and the self-absorption inside the organic medium. The observed photoluminescence peak depends on $\Theta$ according to an outcoupled Bragg mode: $\mathbf{k}_{0} \sin \Theta= \pm \mathbf{k}_{\mathbf{g}} \pm m \mathbf{G}$, where $\mathbf{k}_{0}$ and $\mathbf{k}_{\mathrm{g}}$ are the wavevectors of the free and the guided electromagnetic waves, respectively $\left(\left|\mathbf{k}_{\mathbf{0}}\right|=2 \pi / \lambda\right.$ and $\left.\left|\mathbf{k}_{\mathbf{g}}\right|=2 \pi n_{\text {eff }} / \lambda\right), \mathbf{G}$ is the Bragg vector $(|\mathbf{G}|=2 \pi / \Lambda)$ and $m$ is an integer ${ }^{29}$.

Investigation of the photoluminescence polarization provides further information on the influence of the imprinted grating on fibre emission. We find different angular dispersion for light with $s$ - and $p$-polarization, namely with the electrical field vector parallel or perpendicular to the grating grooves and corresponding to transversal electric (TE) or transversal magnetic (TM) guided modes, respectively (Fig. 4c). The two modes are concomitantly present in the Bragg outcoupled emission, with TM peaks blueshifted by $18-46 \mathrm{meV}$ with respect to the TE peaks, indicating a lower effective refractive index $\left(n_{\text {eff }}\right)$ for light polarized perpendicularly to the nanoimprinted features. In fact, the Bragg coupling condition, stating the conservation of momentum at the nanofibre/air interface, allows us to extract the effective refractive index of the out-fibre coupled guided modes. For an MEH-PPV nanopatterned fibre, $n_{\text {eff }}$ for TM and TE light monotonously decreases from $\sim 1.9$ to 1.2 upon increasing the wavelength from 570 to $575 \mathrm{~nm}$, whereas the difference, $\Delta n_{\text {eff }}=n_{\text {eff,TE }}-n_{\text {eff,TM, }}$, slightly increases from 0.01 to 0.04 (Fig. 4d). For the sake of comparison, we recall that these values are comparable to those previously reported for sub-micrometre period gratings on conjugated polymers and low-molar-mass organic molecules, such as in nanopatterned spiro-terphenyl $\left(n_{\text {eff }}=1.8-1.7 \text { for } 360<\lambda<395 \mathrm{~nm}\right)^{30}$ and MEH-PPV $\left(n_{\text {eff }}=1.7-1.6 \text { for } 579<\lambda<739 \mathrm{~nm}\right)^{31}$ films. 

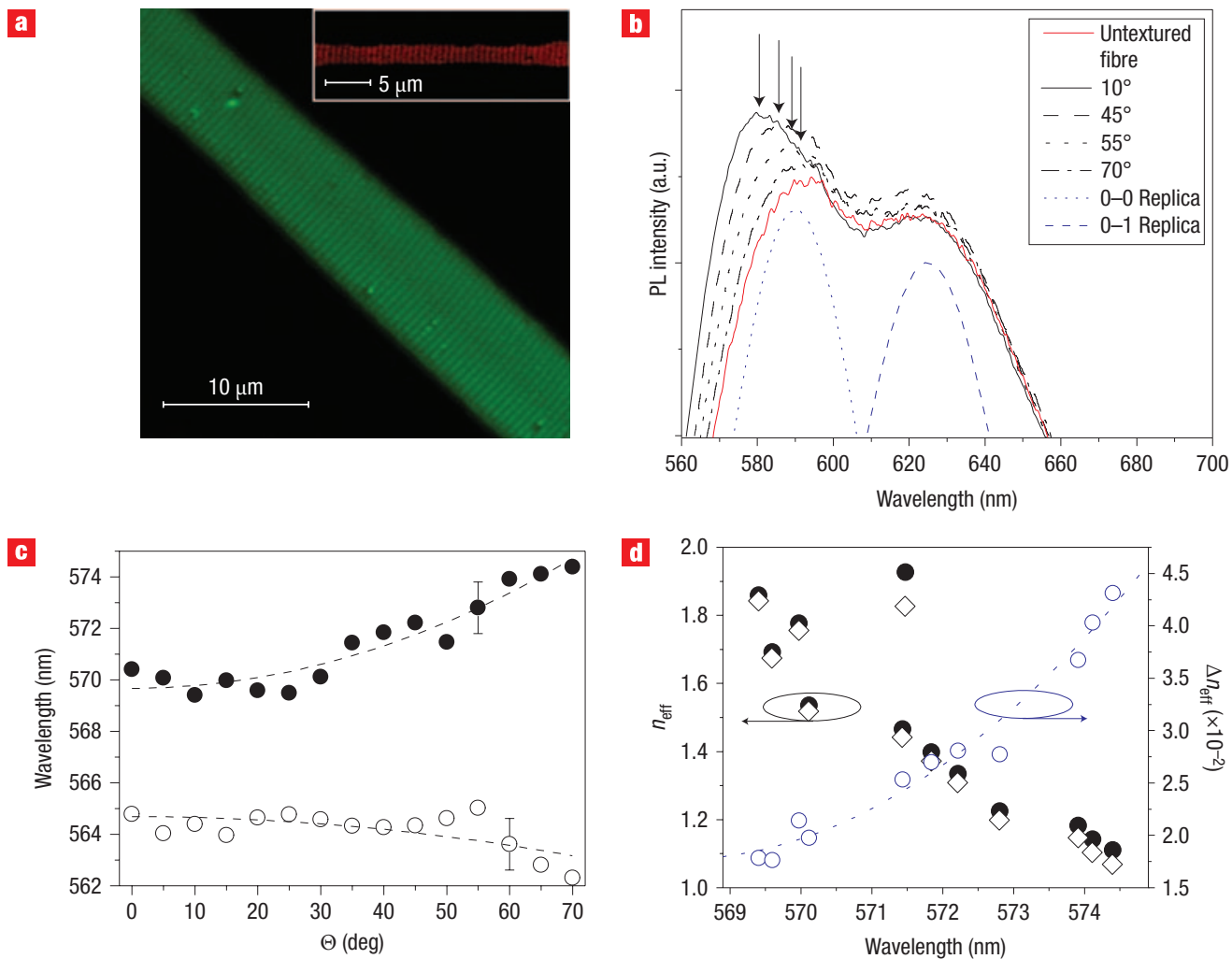

Figure 4 Emission properties of nanopatterned fibres. a, Confocal microscopy pictures of nanoimprinted fibres. The grating period varies from $520 \mathrm{~nm}$ (green GE fibre from 1:8 dimethyl formamide:chloroform solution) to $640 \mathrm{~nm}$ (red MEH-PPV fibre from 1:5 dimethyl formamide:chloroform solution, inset). The GE fibre was imaged under two-photon excitation at $\lambda=800 \mathrm{~nm}$. b. Normalized angle-resolved photoluminescence spectra of an unpatterned and nanoimprinted single MEH-PPV fibre, for various collection angles and TE polarization of the guided mode. The $0-0$ and $0-1$ vibronic replicas of the untextured MEH-PPV fibre, obtained by fitting this spectrum with a Gaussian superposition, are shown as blue curves. The arrows indicate the angular dependence of the emission peaks. c, Angular dispersion of the Bragg-outcoupled modes with $s$ - (that is, for TE guided modes, filled circles) and $p$ - (transverse magnetic (TM), open circles) polarizations. The error bar shown is the same for all points. The lines are guides for the eye. $\mathbf{d}$, Wavelength dependence of the effective refractive index of the Bragg-outcoupled TE (filled circles) and TM (open diamonds) modes of the nanoimprinted fibre, and of the corresponding difference, $\Delta n_{\text {eff }}=n_{\text {eff,TE }}-n_{\text {eff,TM }}$ (open circles). The dotted line is a guide for the eye.

In conclusion, these results indicate that multicolour conjugated polymer nanofibres exhibit remarkable waveguiding properties and can be effectively patterned by room-temperature imprinting. The availability of both full-colour tuneability and flexible patterning technologies, allowing emission enhancement, enables the realization of photonic devices based on conjugated polymer nanofibres such as nanoscale patterned light-emitting diodes and distributed feedback lasers.

\section{METHODS}

The electrostatic spinning setup consisted of a syringe pump (Harvard Apparatus, Holliston, MA) feeding a hypodermic syringe with the polymer solution, an 18-27 gauge stainless steel needle, a high-voltage power supply (XRM30P, Gamma High Voltage Research), and a grounded collector $\left(10 \times 10 \mathrm{~cm}^{2}\right)$ mounted on an isolating stand at an electrostatic spinning distance between 6 and $15 \mathrm{~cm}$. The typical diameter $(S)$ distribution of the fibres that were obtained comprised different populations, labelled A-F in Fig. 1c, where for A, $S<0.65 \bar{S}$; B, $0.65 \bar{S}<S<0.90 \bar{S}$; C, the population of fibres exhibited the most frequent size, $\bar{S}$ (that is, $\bar{S}=380 \mathrm{~nm}$ for the fibres of Fig. 1b); D, 1.4 $\bar{S}<S<1.7 \bar{S}$; E, $S>1.7 \bar{S}$; F, micrometre-scale beads.

For the waveguiding characterization, the fibre photoluminescence was excited by a GaN semiconductor laser diode $(\lambda=405 \mathrm{~nm}$, power $=3 \mathrm{~mW}$, excitation spot diameter $=5 \mu \mathrm{m})$, and detected through a $\times 100$ microscope objective by a colour charge coupled device (CCD) camera. A long-pass filter was used to cut the exciting laser light. Fluorescence micrographs of the emitting fibres were acquired using an upright stereomicroscope equipped with a CCD camera and exciting the nanofibre emission with an Hg lamp. The collected photoluminescence spectra did not vary upon moving from point to point on the same fibre.

The master gratings were fabricated by electron-beam lithography and reactive ion etching on silicon and directly used as a mould for RT-NIL. The spectra of nanopatterned fibres were obtained by collecting the light emitted in a small solid angle $\left(3 \times 10^{-3} \mathrm{srad}\right)$ around each angle value, measured by rotating an optical fibre detector in a plane perpendicular to the surface grating (see Supplementary Information for complete details and the experimental scheme).

Received 5 March 2008; accepted 15 July 2008; published 24 August 2008.

\section{References}

1. Friend, R. H. et al. Electroluminescence in conjugated polymers. Nature 397, 121-128 (1999).

2. Yu, G. et al. Polymer photovoltaic cells: enhanced efficiencies via a network of internal donoracceptor heterojunctions. Science 270, 1789-1791 (1995).

3. Tessler, N., Denton, G. J. \& Friend, R. H. Lasing from conjugated-polymer microcavities. Nature 382, 695-697 (1996)

4. McGehee, M. D. \& Heeger, A. J. Semiconducting (conjugated) polymers as materials for solid-state lasers. Adv. Mater. 12, 1655-1668 (2000).

5. Nguyen, T. -Q. et al. Control of energy transfer in oriented conjugated polymer-mesoporous silica composites. Science 288, 652-656 (2000)

6. Zhang, X., Kale, D. M. \& Jenekhe, S. A. Electroluminescence of multicomponent conjugated polymers. 2. photophysics and enhancement of electroluminescence from blends of polyquinolines. Macromolecules 35, 382-393 (2002).

7. Duan, X. et al. Indium phosphide nanowires as building blocks for nanoscale electronic and optoelectronic devices. Nature 409, 66-69 (2001).

8. Law, M. et al. Nanoribbon waveguides for subwavelength photonics integration. Science 305, 1269-1273 (2004).

9. Menard, E. et al. Micro and nanopatterning techniques for organic electronic and optoelectronic systems. Chem. Rev. 107, 1117-1160 (2007). 
10. Liu, H. et al. Electrospun polymer nanofibres as subwavelength optical waveguides incorporating quantum dots. Small 2, 495-499 (2006).

11. Quochi, F. et al. Gain amplification and lasing properties of individual organic nanofibres. Appl.Phys. Lett. 88, 041106 (2006).

12. Liu, J., Sheina, E., Kowalewski, T. \& McCullough, R. D. Tuning the electrical conductivity and selfassembly of regioregular polythiophene by block copolymerization: nanowire morphologies in new di- and tri-block copolymers. Angew. Chem. Int. Ed. 41, 329-332 (2002).

13. Noy, A. et al. Fabrication of luminescent nanostructures and polymer nanowires using dip-pen nanolithography. Nano Lett. 2, 109-112 (2002).

14. Reneker, D. H. \& Chun, I. Nanometre diameter fibres of polymer, produced by electrospinning Nanotechnology 7, 216-223 (1996).

15. MacDiarmid, A. G. et al. Electrostatically-generated nanofibres of electronic polymers. Synth. Met. $119,27-30$ (2001).

16. Dzenis, Y. Spinning continuous fibres for nanotechnology. Science 304, 1917-1919 (2004).

17. Moran-Mirabal, J. M. et al. Electrospun light-emitting nanofibres. Nano Lett. 7, 458-463 (2007).

18. Kakade, M. et al. Electric field induced orientation of polymer chains in macroscopically aligned electrospun polymer nanofibres. J. Am. Chem. Soc. 129, 2777-2782 (2007).

19. Madhugiri, S. et al. Electrospun MEH-PPV/SBA-15 composite nanofibres using a dual syringe method. J. Am. Chem. Soc. 125, 14531-14538 (2003).

20. Li, D., Babel, A., Jenekhe, S. A. \& Xia, Y. Nanofibres of conjugated polymers prepared by electrospinning with a two-capillary spinneret. Adv. Mater. 16, 2062-2066 (2004).

21. Wei, M., Lee, J., Kang, B. \& Mead, J. Preparation of core-sheath nanofibres from conducting polymer blends. Macromol. Rapid Commun. 26, 1127-1132 (2005).

22. Babel, A., Li, D., Xia, Y. \& Jenekhe, S. A. Electrospun nanofibres of blends of conjugated polymers: morphology, optical properties and field-effect transistors. Macromolecules 38, 4705-4711 (2005).

23. Jang, S. - H. et al. Welded electrochromic conductive polymer nanofibres by electrostatic spinning. Adv. Mater. 17, 2177-2180 (2005).

24. Chronakis, S., Grapenson, S. \& Jakob, A. Conductive polypyrrole nanofibres via electrospinning: Electrical and morphological properties. Polymer 47, 1597-1603 (2006).

25. Lee, K. H. et al. Characterization of nano-structured poly(e-caprolactone) nonwoven mats via electrospinning. Polymer 44, 1287-1294 (2003).
26. Caroll, O. D., Lieberwirth, I. \& Redmond, G. Microcavity effects and optically pumped lasing in single conjugated polymer nanowires. Nature Nanotech. 2, 180-184 (2007).

27. Mele, E. et al. Multilevel, room-temperature nanoimprint lithography for conjugated polymer-based photonics. Nano Lett. 5, 1915-1919 (2005).

28. Hu, D., Yu, J. \& Barbara, P. F. Single-molecule spectroscopy of the conjugated polymer MEH-PPV. J. Am. Chem. Soc. 121, 6936-6937 (1999).

29. Turnbull, G. A. et al. Relationship between photonic band structure and emission characteristics of a polymer distributed feedback laser. Phys. Rev. B 64, 125122 (2001).

30. Spehr, T. et al. Organic solid-state ultraviolet-laser based on spiro-terphenyl. Appl. Phys. Lett. 87, 161103 (2005)

31. Mele, E. et al. Polymeric distributed feedback lasers by room-temperature nanoimprint lithography. Appl. Phys. Lett. 89, 131109 (2006).

Supplementary Information accompanies this paper at www.nature.com/naturenanotechnology.

\section{Acknowledgements}

The authors thank A. Cometta and M. Brich (Zeiss) for the confocal microscopy measurements. F.D.B. acknowledges helpful discussions with F. Ko about the electrostatic spinning technique.

Author contributions

A.C. and D.P. conceived and designed the experiments. F.D.B and S.P. performed the electrospinning experiments. L.P. performed the AFM measurements and analysed the data. R.S. performed the SEM measurements, realized the NIL masters and analysed the data. E.M. carried out the imprinting experiments. A.C. was responsible for the optical characterization. E.M., L.P., A.C., R.C. and D.P. contributed materials and analysis tools. F.D.B. and D.P. co-wrote the paper. All authors discussed the results and commented on the manuscript.

Author information

Reprints and permission information is available online at http://npg.nature.com/reprintsandpermissions/. Correspondence and requests for materials should be addressed to D.P. 